\title{
Analysis of clothing collar structure based on 3D anthropometric measurement
}

\author{
Canyi Huang ${ }^{1, a}$ \\ ${ }^{1}$ Deparment of Business and Information, Quanzhou Normal University, Quanzhou, Fujian, 362000, \\ P.R. China; \\ aemail:yidyj126@sina.com
}

Keywords: 3D anthropometric measurement, clothing collar, structure design, statistic

Abstract. In this paper cross-section shape of human root neck is obtained through 3D anthropometric measurement, and human root neck circumference and the ratio of cervical transverse length and sagittal length are calculated. In addition, through geometric drawing calculation formula as well as the statistical analysis results, each part of clothing collar structure was analyzed and conclude further, and this paper also discuss the feasibility of the formula.

\section{Introduction}

Apparel collar structure is a very important component in apparel design, and we all know the collar is close to the human head, it is the visual center of attention, and it has great influence on the fitness, comfort and aesthetics. The most important part of the collar is called the "basic neck line", which is based on the cross-section shape of human root neck, combined with other characteristics of clothing, and designed as arc-shaped structure line on the upper garment. The basic neck line and the section shape of human neck are in the approximate shape, but sometimes because of the designing need we will change their shape and size, especially in the apparel style structure without collar. As the collar design must take the basic collar line as the basis, so it's necessary to find out principle and calculation methods of collar. This research will access to body size scientifically and accurately, and apply it to clothing collar structure [1].

Three-dimensional body measurement experiment. This experiment uses non-contact 3D laser scanners of Germany TecMath company for human data acquisition, the scanner can scan $2.1 \mathrm{~m}$-high area in $8 \sim 10$ seconds, and the picture resolution can reach $0.5 \mathrm{~mm}$, the measuring accuracy is $\pm 2 \mathrm{~mm}$. Test samples are 500 randomly selected from ages 18 to 25 years old college students , 250 men and 250 women. Measured part is human root neck circumference, measuring method is size from the left side of neck point (SNP), along to anterior cervical point (FNP) over the right side of neck point, to the back neck midpoint (BNP) finally returned to the left side of neck point. This human part is the basis of collar structure design[2]. Figure 1 shows the 3D measurement picture. Figure 2 shows the 3D section of human root neck circumference.

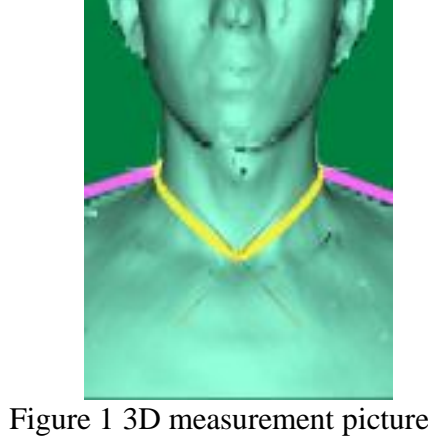

Analysis of human body data descriptive statistics. The measurements obtained 500 human neck circumference data and 500 human root neck section. Neck circumference data automatically report by the research system. In order to have more scientific analysis of human root neck section's 
morphology, we measured the transverse and longitudinal width of the root neck section using system tools additionally. Figure 2 shows the transverse and longitudinal width of the root neck section. And then a new derived variable neck transverse sagittal ratio, which is calculated by the root neck transverse width / longitudinal wide of root neck section. Table 1 shows the descriptive statistics results from SPSS17.0 statistical analysis software. We can see from the table, the average of root neck circumference is about $38 \mathrm{~cm}$, ranges from $34.3-44.1 \mathrm{~cm}$; the average transverse sagittal ratio is 1.21 , so we can see that human neck root transverse width is slightly larger than the longitudinal width[3,4].

Table 1 neck rhizosphere descriptive statistics and the transverse cervical sagittal ratio analysis results

\begin{tabular}{c|c|c|c|c|c}
\hline parts & Sample & minimum & maximum & mean & STD \\
\hline $\begin{array}{c}\text { Root neck } \\
\text { circumference }\end{array}$ & 500 & 34.3 & 44.1 & 38.313 & 2.1123 \\
Neck transverse & 500 & 1.03 & 1.33 & 1.21 & 0.058 \\
sagittal ratio & & & & \\
\hline
\end{tabular}

\section{Calculation method of collar structure and the effectiveness demonstration.}

Calculation method of collar structure. There are two controlling parts in the structure design of collar, "horizontal neck" and "vertical neck", horizontal neck refers to the transverse diameter of collar circle, vertical neck refers to the longitudinal diameter of collar circle. Both lengths can be calculated by the formula according to the human body and neck size [5, 6].

First we draw a circle referring to the statistical results: the average collar circumference $38.3 \mathrm{~cm}$, and then according to the extracted human body's 3D neck section shape, divide the circle's diameter CD five equal parts, Take $\mathrm{OC}_{1}=1 / 5 \mathrm{CD}$, and determine the dot $\mathrm{C}_{1}$, we define $\mathrm{OC}_{1}$ as back vertical neck, then take $\mathrm{DD}_{1}=\mathrm{OC}_{1}=1 / 5 \mathrm{CD}$, determine the dot $\mathrm{D}_{1}$, we define $\mathrm{OD}_{1}$ as back horizontal neck. We can see from figure 3. After adjusting, Longitudinal distance of the collar $\mathrm{C}_{1} \mathrm{D}_{1}$ less half of $\mathrm{DD}_{1}$ compare to round's diameter $\mathrm{CD}$, in order to make the circumferential length be equal before and after adjustment, transverse diameter $\mathrm{AB}$ should corresponding increase the length by half of $\mathrm{DD}_{1}$, which increases $\mathrm{AA}_{1}=1 / 4 \mathrm{DD}_{1}, \mathrm{BB}_{1}=1 / 4 \mathrm{DD}_{1}, \mathrm{OB}_{1}$ or $\mathrm{OA}_{1}$ is defined as the horizontal neck [7].Fig 3 and Fig4 show the details.

Then draw out the basic collar shape by going though four control points $A_{1} B_{1} C_{1} D_{1}$, using the measurement function of the clothing $\mathrm{CAD}$ we get " $\mathrm{AA}_{1}=\mathrm{BB}_{1}=0.6$ ", and the circumference measured after the adjustment is still $38.3 \mathrm{~cm}$, that means a round adjusted into irregular section shape of ellipse follow the neck shape of human body, its circumference remained the original specifications.

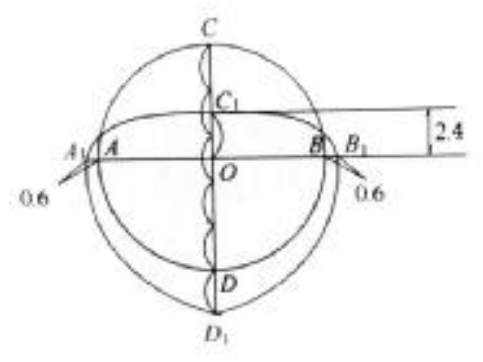

Fig. 3 collar geometry deformation

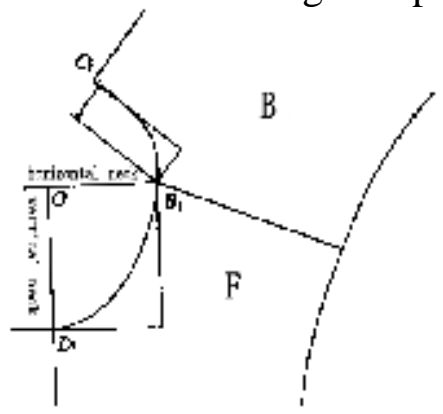

Fig. 4 collar structure chart

We will make further confirmation combining with drawing: 
Circle's diameter $\mathrm{AB}=\mathrm{CD}=38 \mathrm{~cm} / \Pi \approx 12.2$

Back vertical neck $\mathrm{OC}_{1}=1 / 5 \mathrm{CD}=12.2 / 5 \approx 2.4$

Front horizontal neck $=\mathrm{OB}_{1}=\mathrm{OB}+\mathrm{BB}_{1}=$ collar circumference $/ 2 \Pi+0.6 \approx 0.16$ collar circumference $+0.6$

Front vertical neck $\mathrm{OD}_{1}=\mathrm{OD}+\mathrm{DD}_{1} \approx 0.16$ collar circumference +2.4

In order to facilitate the application and calculation of the formula, the 0.16 collar circumference is adjusted to 1/6 collar circumference, the difference before and after adjustment for the two formula is: $1 / 6$ collar circumference -0.16 collar circumference $=1 / 150$ collar circumference; according to the average collar men and women $38.3 \mathrm{~cm}$, the error value before and after adjusting is $38.3 * 1 / 150=0.3$, take the error value as correction value, the calculation formula after correcting:

Front horizontal neck $=1 / 6$ collar circumference +0.3

Back horizontal neck $=1 / 6$ collar circumference +0.3

Front vertical neck $=1 / 6$ collar circumference +2.1

Back vertical neck $=2.4$

Validation of effectiveness. Although these formulas are approximate, and as the numerical changes of human root neck circumference is small, we can see from the statistics table 1 that the value changes between $34.3-44.1 \mathrm{~cm}$, the maximum difference with intermediate body $38 \mathrm{~cm}$ is only $6.1 \mathrm{~cm}$. According to formula to calculate the error $1 / 150$, the maximum error is only $0.04 \mathrm{~cm}$; the error value is far lower than the garment tolerance standards stipulated by the state, so the formula is feasible and effective.

\section{Conclusion}

The clothing structure should take human as the basis, this research uses3D measurement for human root neck circumference and cross-sectional morphology of root neck, and takes statistical analysis of data obtained, then combines with the actual drawing on the collar structure of geometric analysis, finally obtained the calculating formula of garment collar structure, and further demonstrates that the formula is feasible and effective.

\section{Acknowledgements}

This work was financially supported by the Education and scientific research projects of young and middle-aged teachers in Fujian Province (JA15391).

\section{References}

[1] DC.Wu, XJ.Gu. Analysis on the characteristics of apparel's mass customization. Journal of textile, 2005.3,p.78.

[2] CY.Huang, X.Zhang. Study on neck clustering and dimensions specification for young man. Journal of textile, 2007.1,p.121.

[3] H.Dai , "Apparel size standard system and application," press of china textile 2001,P.10-11.

[4] The National Standard GB/T1335.1-1997 apparel size - men, State Bureau of Technical Supervision, 1997:11-13.

[5] JM .Su. Application and development guide of statistical software SPSS 17.0 for windows, press of electronic industry 2004,P.315. 
[6] B.Pan . Industrial apparel pattern design. Beijing: China textile press, 2000,P.18-35.

[7] WD.Lu , SPSS for Windows statistical analysis. Beijing Publishing House of Electronics Industry, 2002.P.141. 